majority preferred the sexual health clinic as an acceptable venue $37 \%$ (56/150). A weekly "in-reach" service was set up with the local Drug Service to run alongside the MSM evening clinic. From August to December 2014, there were 15 clinics in total with 21 visits (max capacity 30 visits). $25 \%$ of those seen were from the local borough; the rest of the clients were from neighbouring boroughs.

Discussion/conclusion The service to date has been a clinical and operational success. A patient satisfaction questionnaire completed by 13 clients noted $92 \%$ were happy to be seen at this venue, $85 \%$ felt the provision of this service was worthwhile and $85 \%$ would recommend this service to others. Further work in this area with a targeted MSM history proforma, chemsex leaflet and needle exchange schemes are also being developed.

\section{P146 ESTABLISHING A SEXUAL HEALTH RESEARCH PRACTICE NETWORK IN THE NORTH EAST}

${ }^{1}$ Kirsty Foster*, ${ }^{1}$ Claire Sullivan, ${ }^{2}$ Mandy Cheetham, ${ }^{2}$ Janet Shucksmith, ${ }^{3}$ Anne McNall, ${ }^{4}$ Lynn Wilson, ${ }^{5}$ Sarah Duncan. ${ }^{1}$ Public Health England, Newcastle Upon Tyne, UK; ${ }^{2}$ Teesside University, Middleborough, UK; ${ }^{3}$ Northumbria University, Newcastle Upon Tyne, UK; ${ }^{4}$ Durham County Council, Durham, UK; ${ }^{5}$ County Durham and Darlington NHS FT, Darlington, UK

\subsection{6/sextrans-2015-052126.189}

Background/introduction There is a strong tradition of collaborative research and practice in sexual health in the North East of England.

Aim(s)/objectives The North East Sexual Health Research Practice Network brings together colleagues from academia, public health and clinical practice to share research findings and identify research questions based on local issues.

Methods A project group with representatives from local universities, Public Health England and local authorities developed a proposal for a regional sexual health research network to promote collaboration and share evidence of what works. A steering group was established to develop an initial work plan for the network.

Results The network has identified key outputs for its first year -including a website hosted by FUSE (the Centre for Translational Research in Public Health, a collaboration between the five North East universities), a mapping exercise of existing sexual health research in the region and an inaugural Research Practice event to share key findings and plan future projects.

Discussion/conclusion We have identified an enthusiasm for sexual health research in the region, and hope that the network will draw together colleagues working in different fields who may not be aware of the range of work being carried out across the region. We hope that by identifying research questions that are locally meaningful, and by offering support from colleagues with expertise in the field, we will generate research that will inform sexual health practice and commissioning, reduce duplication and ultimately improve the sexual health of people in the North East and beyond.

\section{P147 A TRUST-WIDE AUDIT ON PELVIC INFLAMMATORY DISEASE MANAGEMENT IN A GENITOURINARY MEDICINE SETTING}

Rebecca Marcus*, Mary Tiab, Sarah Teague, Gerard Gorman, Mags Portman, Liat Sarner. Barts Health, London, UK

10.1136/sextrans-2015-052126.190
Background/introduction Diagnosis and management of pelvic inflammatory disease (PID) in the genitourinary medicine clinic can be challenging. Optimising management is essential in preventing potential sequelae. The national BASHH PID audit (2012) indicated that adherence to guidelines was inconsistent.

Aim(s)/objectives To audit PID management to help inform introduction of new trust guidelines.

Methods Retrospective case note review of all patients with a PID clinic code over six months at three clinics across the trust.

Results Of 184 cases identified, 99.5\% of patients had either one or more of PID symptoms: lower abdominal pain, dyspareunia, abnormal bleeding, vaginal discharge. $92 \%$ and $97.8 \%$ of patients underwent microscopy and STI screening respectively. 16 tested positive for chlamydia, 4 for gonorrhoea, 5 for herpes simplex virus, 2 for trichomonas vaginalis, 47 for bacterial vaginosis (BV), 8 for urinary tract infection (UTI) and 10 for candida. $61 \%$ received a recommended treatment regimen, with up to 20 different treatment regimens prescribed. $44 \%$ of patients attended for follow-up after two weeks.

Discussion/conclusion In this cohort, there were relatively few STI diagnoses, with BV being the most likely microbiological diagnosis. There was wide variation in prescribing practice and adherence to local and national guidelines. Diagnostic criteria for PID were simplified and disseminated at a trust-wide meeting. New trust guidelines were introduced taking local resistance patterns and national guidance into account.

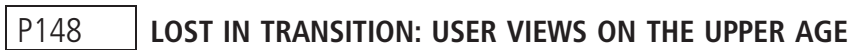 LIMIT IN ACCESSING CONTRACEPTION AND SEXUAL HEALTH SERVICES}

Kimberley Forbes, Arshia Tavender, Elizabeth Okecha*, David Daniels, Richard West. Sexual Health Hounslow, London, UK

\subsection{6/sextrans-2015-052126.191}

Background/introduction In 2008 Integrated Contraception and Sexual Health (CASH) Services for those under 25s were launched at community and level-three sites. The age cap of 25 was linked to Chlamydia screening targets.

Aim(s)/objectives Staff highlighted concern regarding older clients and young people under 18 accessing services simultaneously. It was decided to consult user views before changes were made.

Methods Questionnaires were given to those under 25 attending CASH and level-three sites with choice regarding service access and age limit, 18, 20 or 25 and whether they had attended during a dedicated YP session.

Results 295 respondents; 41 male (13.9\%). 2/14 <16s (14\%), $9 / 57<18$ s $(16 \%)$, no $18-19$ years olds and $10 / 156>20$ s $(6.4 \%)$ identified as attending during a dedicated YP clinic. 9/15<16s (60\%), $41 / 58$ of $<18$ s (71\%), 52/66 18-19 years old (79\%) and $125 / 156$ of $>20$ s $(79 \%)$ preferred the age limit of 25 .

Discussion/conclusion Surprisingly the majority of respondents from all age groups preferred 25 to be the maximum age for young people's CASH services. A small number of respondents were under 16 and further work with younger clients to address hidden concerns may be indicated. Older YP still preferred YPorientated sessions however the majority of respondents attended out of dedicated young people session times highlighting the need for mainstream services to offer a young people friendly service during all sessions. 